\title{
Influential Article Review - What Are the Information System Competencies That Managers Must Have? Advancement of Public Administration Degree Program
}

\author{
Aleeza Townsend \\ Dolcie Callahan \\ Waqar Coffey
}

This paper examines information technology. We present insights from a highly influential paper. Here are the highlights from this paper: This paper analyzes the competences required by executives to manage information system, and consequently, the competences that must define the information system subjects in non-technical degrees, degrees, such as Public Administration or Business Management. This work reviews the literature about business managers' competences on Information Technologies (IT) and compares the theory with the traditional body of knowledge about information systems taught at business schools. By analyzing the executives' function, their role in the information system management, and, above, all the importance of their decisions in the effective integration of IT in business processes, this work proposes specific development in seven knowledge areas that facilitate the acquisition of these types of executive competences. For our overseas readers, we then present the insights from this paper in Spanish, French, Portuguese, and German.

Keywords: information systems, competences, managers, management degree, public administration studies

\section{SUMMARY}

- Within the MIS literature, there are numerous works that study the role of top managers in the efficiency of the IS in organizations. This study is mainly based on the exceptional paper of Bassellier et al. on the subject and tries to update the proposal of these authors and apply it to the development of business schools' curricula.

- Since the outset of IS, the importance of the executives' support has been recognized to obtain all the benefits which IT systems can offer. One of the first studies about the matter showed that the managers who participate in the development of IS achieve a greater appreciation of the system. This means that the participation has an influence on the appreciation. Swanson describes the «appreciation» concept as the executive's belief about the relative value of IS in a particular business activity. 
- It is in the mid-eighties when IT ceased to be confined to the specific aspects of the business activity and was used on a generalized basis in the organization, affecting the way of doing business in a substantial way. Here, the strategic vision became necessary to guide their development and the general managers' participation was mandatory. This led certain authors to state that IS are too important to be abandoned in the hands of the technicians. Then, since the outset of the extensive IT use in organizations, the involvement of executives in IS has been considered as necessary to exploit the advantages, which these technologies offer. A reference work of this period is that of Jarvenpaa and Ives. These authors analyzed the important role managers play in the decisions of how, when, and where to use the IT resources. The results found a positive correlation between the involvement, participation, age, and professional training of the executives on IT, on the one hand, and the progressive use of IT in the company, on the other hand. This same study showed that the involvement of the executives in the activities linked to the IS area is positively correlated to the participation of executives in the organizational planning of IS, and yet, weakly associated with the executive's functional training on IT.

- Boyatzis defines the managers' IT knowledge as specialized knowledge, a useful body of facts and relevant concepts for a specific job. Based on the Boyatzis' definition, Bassellier et al. consider three major knowledge areas that managers must possess to exploit IT in the organization: Keep up to date with the information assets and the information opportunities. Understand the value and potential of IT. Know the potential as well as current and future limitations of IT and determine how rival companies use them.

- Another classification of the required IT know-how is provided by Brown and Magill. In this case, the authors suggest a division between the management of the technology specifically and the management of its application in the information systems: Technology management. Technology use management.

- Other works consider that the main purpose of managers IT knowledge is to permit the executives to communicate with the IT personnel. This knowledge facilitates the «link» or the connection between the business system, and the IS Department.

- First, it is necessary to make the distinction, as suggested by Sabherwal and Chan, between the IT strategy, the IS strategy, and the information management strategy. The IS strategy is focused on the IT business systems or applications, whose main objective is their alignment with the business requirements and their use to achieve strategic benefits. The IT strategies are focused on the technology policies, including such aspects as the architecture, technical standards, security levels, and risk attitudes. Finally, the information management strategies are concerned with the structures and functions for the IS and IT administration, focusing on issues, such as the relations between experts and users, the managers' responsibilities, the management controls, and the performance measurement processes. In this study, when we refer to the IT strategy, we include the applications administration, and hence, we also encompass the IS strategies.

\section{HIGHLY INFLUENTIAL ARTICLE}

We used the following article as a basis of our evaluation:

Devece Carañana, C., Peris-Ortiz, M., \& Rueda-Armengot, C. (2016). What are the competences in information system required by managers? Curriculum development for management and public administration degrees. Technology, Innovation and Education, 2(1).

This is the link to the publisher's website:

https://technology-innovation-education.springeropen.com/articles/10.1186/s40660-016-0016-2 


\section{INTRODUCTION}

The Bologna process requires the definition of the objectives to be achieved in a subject in terms of knowledge, skills, and competences. This requires in-depth thought about the competences, described in terms of knowledge, skills, attitudes, and abilities, in which the graduates must acquire to successfully handle their work, both in their daily tasks as well as their professional development throughout their career. To develop these competences is the final object of an educational program (European Commission 2015).

According to the European Commission (2015), competence can be defined as the proven ability to use knowledge, skills and personal, social and/or methodological abilities, in work or study situations, and in professional and personal development.

Competences are subject specific or generic. This paper aims to explore the subject-specific competences, regarding the information system subjects which are taught in management degrees. These degrees include Business Management and Public Administration. The objective of these degrees is to train managers (executives) capable of assuming responsibility within the organizations for which they work. Consequently, this paper begins by defining the concept of executive competence in a generic way, subsequently, developing these executive competences in detail in relation to information systems.

\section{Executive Competences}

From a business perspective, competence is the ability to sustain the coordinated deployment of assets in ways that help a firm to achieve its goals (Sanchez et al. 1996).

Executive competences are essential, since they allow to interpret the environment and to create a unique vision based on which it is effectively possible in business. This vision or mental model (Senge 1990) of the organization and the environment makes possible to detect opportunities and threats, establishing the organization's strategic approach to exploit asymmetries in markets with strategic factors (Lado and Wilson 1994; Hambrick and Mason 1984). Executives are the persons in charge of setting the mission and objectives that guide the creation and implementation of strategy, and the development of organizational skills and capabilities (Lado et al. 1992).

The executive competences originate from both explicit and tacit knowledge, based on the executives' experience. The executive competences usually include both the abilities and know-how of the executives on an individual basis, as well as the skills and capacities derived from the interaction with other staff members.

Leadership is also considered as a determining success factor for managers. Leadership allows to communicate the mission and to obtain the commitment from the entire organization, thus allowing its members to act in a collective rather than isolated way (Lado and Wilson 1994; Lado et al. 1992; Westley and Mintzberg 1989). Osbaldeston and Barham (1992) insist on this point, indicating the link of the company's competitiveness with the management competence to develop and completely use the experience and talent of all the members of the organization, integrating and encouraging the efforts of a complex team of persons. Thus, management competences cover aspects as diverse as general and specialized knowledge, individual abilities as well as social ones, organization and planning skills, capacity to innovate, and cope with non-routine activities.

These types of competences decisively determine the acquisition, development and deployment of the firm's resources and capacities, their conversion into valuable products, and the creation of value. Thus, Penrose (1959), Hambrick and Brandon (1988), and Castanias and Helfat (1991) emphasize the value of executive talent and its heterogeneity to improve the company's competitive rank. More recently, Bettiol et al. (2012) confirm the value of the managers' unique vision to interpret the environment, and the importance of this vision to exploit the firm's resources (Yang and Li 2011; Siegel and Renko 2012).

Researchers differ in relation to the dimensions that comprise the executive competences. For example, Lado and Wilson (1994: 703) solely distinguish two dimensions: the outline of a strategic vision and the establishment of a beneficial link with the environment. Lado et al. (1992), however, identify a third dimension, leadership, independently of the dimension of the strategic vision. Camisón (2002) integrates these models, proposing five dimensions in the executive competences: (1) Strategic approach. The 
strategic approach is the executive capability to outline a strategic vision that supports the development of the organizational resources and establish a beneficial organization-environment link. (2) Qualification of the executives: This includes their talent, technical and management knowledge, their ability to solve problems, and make decisions and their experience. (3) Personality of the executives. This dimension alludes to the executives' knowledge about themselves, their capability to control their own behavior and emotions, and the values and standards that motivate them, which condition their social model and conduct. (4) Executive mentality. Orientation of the managers in relation to achievement, time use, change and innovation, as well as risk (revealing their perspective when facing risk). (5) Effective leadership. This alludes to the management style and leadership ability to communicate the strategic vision (a clear vision of the organization's future) to the members of the organization and to delegate power to the employees to carry it out, achieving their enthusiastic commitment.

In our study, we are interested in the direct effects of the executive competences in information systems (IS). For this purpose, we focused on the specific aspects of the executive competences in relation to the information management in organizations, limiting our study to the executive competences in IS which are described below.

\section{CONCLUSION}

This study analyzes the competences that a manager should acquire about information systems. This paper clearly shows the need for middle or top managers to achieve these competences to achieve the competitive edge in the organizations they work for. One of the most important functions of these competences is the possibility to create a vision of IT and how these technologies can be applied to organizations. Always bearing this strategic vision in mind, the information system courses for managers must develop seven essential competences. The first is knowledge in information technologies. The second competence is linked to the knowledge of the existing applications in the market and which programs exist for each of the specific functions of the organization and how they can provide support to the activities and to the decision-making processes, as well as the information systems as the basic coordination component among activities. Third, it is necessary to create competences in the development and analysis of information systems, linked to IS creation or upgrade projects in the organizations. The fourth competence involves the acquisition of abilities in IS administration and all the aspects linked to security and audits. Fifth, e-commerce, web 2.0, and the new tendencies in mobile technologies and applications should be included in the curricula of the IS subjects. Sixth, managers must know about the legal aspect of data protection, e-commerce, and e-government. Finally, although with a less academic nature, it is necessary to encourage students about the importance of knowing reliable IT experts and consultants who are vitally important for SME. This basic framework of competences with the proposed strategic approach should appear in the subjects of information management in non-technical degrees with a management profile.

\section{REFERENCES}

Bassellier, G., \& Benbasat, I. (2004). Business competence of IT professionals: Conceptual development and influence on IT-business partnerships. MIS Q, 28(4), 673-694.

Bassellier, G., Benbasat, I., \& Reich, B.H. (2003). The influence of business managers' IT competence on championing IT. Inf Syst Res, 14(4), 317-336.

Bassellier, G., Reich, B.H., \& Benbasat, I. (2001). Information technology competence of business managers: A definition and research model. J Manag Inf Syst, 17(4), 159-182.

Bettiol, M., Di Maria, E., \& Finotto, V. (2012). Marketing in SMEs: The role of entrepreneurial sensemaking. Int Entrep Manag J, 8(2), 223-248.

Boyatzis, R.E. (1982). The competent manager a model for effective performance. Wiley, New York.

Boynton, A.C., Zmud, R.W., \& Jacobs, G.C. (1994). The influence of IT management practice on IT use in large organizations. MIS Q, 18(3), 299-318. 
Broadbent, M., \& Weill, P. (1993). Improving business and information strategy alignment: Learning from the banking industry. IBM Syst J, 32(1), 162-179.

Brown, C.V., \& Magill, S.L. (1994). Alignment of the IS functions with the enterprise: Toward a model of antecedents. MIS Q, 18(4), 371-403.

Busch, E.A., Jarvenpaa, S.L., Tractinsky, N., Glick, W.H. (1991). External versus internal perspectives in determining a firm's progressive use of information technology. Proceedings of the 12th International Conference on Information Systems, pp. 239-250. New York.

Camisón, C. (2002). On the relevance of industry, competitive scope, strategic group, size and distinctive competences construct on explaining of organizational performance. Universitat jaume I, Castellón [Working Paper 1-02,Research Group on Strategy, Knowledge Management and Organizational Learning].

Castanias, R.P., \& Helfat, C.E. (1991). Managerial resources and rents. J Manag, 17, 155-171.

Cegarra-Navarro, J.G., Sánchez-Vidal, M.E., \& Cegarra-Leiva, D. (2011). Balancing exploration and exploitation of knowledge through an unlearning context: an empirical investigation in SMEs. Manag Decis, 49(7), 1099-1119.

Chan, Y.E., \& Reich, B.H. (2007). IT alignment: What have we learned? J Inf Tech, 22, 297-315.

Chan, Y.E., Sabherwal, R., \& Thatcher, J.B. (2006). Antecedents and outcomes of strategic IS alignment: An empirical investigation. IEEE Trans Eng Manag, 51(3), 27-47.

Croteau, A.M., \& Bergeron, F. (2001). An information technology trilogy: Business strategy, technological deployment and organizational performance. J Strateg Inf Syst, 10, 77-99.

Crowston, K., \& Myers, M.D. (2004). Information technology and the transformation of industries: Three research perspectives. J Strateg Inf Syst, 13(1), 5-28.

Dent-Micallef, A., \& Powell, T. (1998). Technologies de l'information: Nécessités stratégiques ou sources d'avantage concurrentiel? Une étude empirique dans le secteur de la distribution aux Etats-Unis. Rev Can Sci Adm, 15(1), 39-64.

Devece, C. (2013). The value of business managers' 'information technology' competence. Serv Ind J, $33(7 / 8), 720-733$.

Doll, W.J., \& Torkzadeh, G. (1987). The relationship of MIS steering committee to size of firm and formalization of mis planning. Commun ACM, 30(11), 972-978.

Earl, M.J. (1996). The risks of outsourcing IT. Sloan Manag Rev, 37(3), 26-32.

European Commission. (2015). ECTS Users' Guide 2015. Publications Office of the European Union, Luxembourg. doi:10.2766/87592

Gao, W., Li, Q., \& Drougas, A. (2017). Ownership Structure and Stock Price Crash Risk: Evidence from China. Journal of Applied Business and Economics, 19(4). Retrieved from https://articlegateway.com/index.php/JABE/article/view/715

Hambrick, D.C., \& Brandon, G. (1988). Executive values. In D.C. Hambrick (Ed.), The executive effect: concepts and methods for studying top managers. JAI Press, Greenwich.

Hambrick, D.C., \& Mason, P.A. (1984). Upper echelons: The organization as a reflection of its top managers. Acad Manag Rev, 9(2), 193-206.

Hotho, S., \& Champion, K. (2011). Small businesses in the new creative industries: Innovation as a people management challenge. Manag Decis, 49(1), 29-54.

Jarvenpaa, S.L., \& Ives, B. (1991). Executive involvement and participation in the management of IT. MIS Q, 15(2), 52-69.

Lado, A.A., Boyd, N.G., \& Wright, P. (1992). A competency-based model of sustainable competitive advantage: Toward a conceptual integration. J Manag, 18(1), 77-91.

Lado, A.A., \& Wilson, M.C. (1994). Human resource systems and sustained competitive advantage: a competency-based perspective. Acad Manag Rev, 19(4), 699-727.

Lederer, A.L., \& Mendelow, A.L. (1988). Information systems planning: Top management takes control. Bus Horiz, 31(3), 73-78.

Oh, W., \& Pinsonneault, A. (2007). On the assessment of the strategic value of information technologies: conceptual and analytical approaches. MIS Q, 31(2), 239-265. 
Osbaldeston, M., \& Barham, K. (1992). Using management development for competitive advantage. Long Range Plan, 25(6), 18-24.

Penrose, E.T. (1959). The theory of the growth of the firm. Basil Blackwell, Oxford.

Reich, B.H., \& Benbasat, I. (1996). Measuring the linkage between business and information technology objectives. MIS Q, 20(1), 55-81.

Reich, B.H., \& Benbasat, I. (2000). Factors that influence the social dimension of alignment between business and information technology objectives. MIS Q, 24(1), 81-113.

Riegner, C. (2007). Word of mouth on the web: the impact of web 2.0 on consumer purchase decisions. $J$ Advert Res, 47(4), 436-447.

Rockart, J.F. (1988). The lines takes the leadership. Sloan Manag Rev, 29(4), 57-64.

Sabherwal, R., \& Chan, Y.E. (2001). Alignment between business and IS strategies: A study of prospectors, analyzers, and defenders. Inf Syst Res, 12(1), 11-33.

Sanchez, R., Heene, A., \& Thomas, H. (1996). Towards the theory and practice of competence-based competition. In R. Sanchez, A. Heene, \& H. Thomas (Eds.), Dynamics of competence-based competition: theory and practice in the new strategic management (pp 1-35). Elsevier, London.

Senge, P.M. (1990). The fifth discipline: The age and practice of the learning organization. Century Business, London.

Siegel, D.S., \& Renko, M. (2012). The role of market and technological knowledge in recognizing entrepreneurial opportunities. Manag Decis, 50(5), 797-816.

Stare, M., Jaklic, A., \& Kotnik, P. (2006). Exploiting ICT potential in service firms in transition economies. Serv Ind J, 26(3), 287-302.

Swanson, E.B. (1974). Management information systems: appreciation and involvement. Manag Sci, $21(2), 178-188$.

Torkzadeh, G., \& Xia, W. (1992). Managing telecommunications by steering committee. MIS Q, 16(2), 187-199.

Westley, F., \& Mintzberg, H. (1989). Visionary leadership and strategic management. Strateg Manag J, $10,17-32$.

Yang, T.T., \& Li ,C.R. (2011). Competence exploration and exploitation in new product development: The moderating effects of environmental dynamism and competitiveness. Manag Decis, 49(9), $1444-1470$.

\section{TRANSLATED VERSION: SPANISH}

Below is a rough translation of the insights presented above. This was done to give a general understanding of the ideas presented in the paper. Please excuse any grammatical mistakes and do not hold the original authors responsible for these mistakes.

\section{VERSION TRADUCIDA: ESPAÑOL}

A continuación se muestra una traducción aproximada de las ideas presentadas anteriormente. Esto se hizo para dar una comprensión general de las ideas presentadas en el documento. Por favor, disculpe cualquier error gramatical y no responsabilite a los autores originales de estos errores.

\section{INTRODUCCIÓN}

El proceso de Bolonia requiere la definición de los objetivos a alcanzar en una asignatura en términos de conocimientos, habilidades y competencias. Esto requiere un pensamiento profundo sobre las competencias, descritas en términos de conocimientos, habilidades, actitudes y habilidades, en las que los graduados deben adquirir para manejar con éxito su trabajo, tanto en sus tareas diarias como en su desarrollo profesional a lo largo de su carrera. Desarrollar estas competencias es el objeto final de un programa educativo (Comisión Europea 2015). 
Según la Comisión Europea (2015), la competencia puede definirse como la capacidad demostrada para utilizar conocimientos, habilidades y habilidades personales, sociales y/o metodológicas, en situaciones de trabajo o estudio, y en el desarrollo profesional y personal.

Las competencias son específicas de la asignatura o genéricas. Este trabajo tiene como objetivo explorar las competencias específicas de la asignatura, con respecto a las asignaturas del sistema de información que se imparten en los grados de gestión. Estos grados incluyen Administración de Empresas y Administración Pública. El objetivo de estas titulaciones es formar directivos (ejecutivos) capaces de asumir responsabilidades dentro de las organizaciones para las que trabajan. En consecuencia, este trabajo comienza definiendo el concepto de competencia ejecutiva de manera genérica, desarrollando posteriormente estas competencias ejecutivas en detalle en relación con los sistemas de información.

\section{Competencias Ejecutivas}

Desde una perspectiva empresarial, la competencia es la capacidad de sostener el despliegue coordinado de activos de manera que ayude a una empresa a alcanzar sus objetivos (Sánchez et al. 1996).

Las competencias ejecutivas son esenciales, ya que permiten interpretar el entorno y crear una visión única en base a la cual es efectivamente posible en los negocios. Esta visión o modelo mental (Senge 1990) de la organización y el entorno permite detectar oportunidades y amenazas, estableciendo el enfoque estratégico de la organización para explotar asimetrías en mercados con factores estratégicos (Lado y Wilson 1994; Hambrick y Mason 1984). Los ejecutivos son las personas encargadas de establecer la misión y los objetivos que guían la creación e implementación de la estrategia, y el desarrollo de habilidades y capacidades organizacionales (Lado et al. 1992).

Las competencias ejecutivas se originan en el conocimiento explícito y tácito, basado en la experiencia de los ejecutivos. Las competencias ejecutivas suelen incluir tanto las habilidades y conocimientos técnicos de los ejecutivos de forma individual, como las habilidades y capacidades derivadas de la interacción con otros miembros del personal.

El liderazgo también se considera como un factor determinante de éxito para los gerentes. El liderazgo permite comunicar la misión y obtener el compromiso de toda la organización, permitiendo así a sus miembros actuar de manera colectiva en lugar de aislada (Lado y Wilson 1994; Lado et al. 1992; Westley y Mintzberg 1989). Osbaldeston y Barham (1992) insisten en este punto, indicando el vínculo de la competitividad de la empresa con la competencia de gestión para desarrollar y utilizar completamente la experiencia y el talento de todos los miembros de la organización, integrando y fomentando los esfuerzos de un complejo equipo de personas. Así, las competencias de gestión abarcan aspectos tan diversos como el conocimiento general y especializado, las capacidades individuales y sociales, las habilidades de organización y planificación, la capacidad de innovar y hacer frente a actividades no rutinarias.

Este tipo de competencias determinan decisivamente la adquisición, el desarrollo y el despliegue de los recursos y capacidades de la empresa, su conversión en productos valiosos y la creación de valor. Así, Penrose (1959), Hambrick y Brandon (1988), y Castanias y Helfat (1991) enfatizan el valor del talento ejecutivo y su heterogeneidad para mejorar el rango competitivo de la empresa. Más recientemente, Bettiol et al. (2012) confirman el valor de la visión única de los gerentes para interpretar el medio ambiente, y la importancia de esta visión para explotar los recursos de la empresa (Yang y Li 2011; Siegel y Renko 2012).

Los investigadores difieren en relación con las dimensiones que comprenden las competencias ejecutivas. Por ejemplo, Lado y Wilson (1994: 703) distinguen únicamente dos dimensiones: el esquema de una visión estratégica y el establecimiento de un vínculo beneficioso con el medio ambiente. Lado et al. (1992), sin embargo, identifican una tercera dimensión, el liderazgo, independientemente de la dimensión de la visión estratégica. Camisón (2002) integra estos modelos, proponiendo cinco dimensiones en las competencias ejecutivas: (1) Enfoque estratégico. El enfoque estratégico es la capacidad ejecutiva para delinear una visión estratégica que apoye el desarrollo de los recursos de la organización y establecer un vínculo beneficioso entre la organización y el entorno. (2) Calificación de los ejecutivos: Esto incluye su talento, conocimientos técnicos y de gestión, su capacidad para resolver problemas y tomar decisiones y su experiencia. (3) Personalidad de los ejecutivos. Esta dimensión alude al conocimiento de los ejecutivos sobre sí mismos, su capacidad para controlar su propio comportamiento y emociones, y los valores y 
estándares que los motivan, que condicionan su modelo social y conducta. (4) Mentalidad ejecutiva. Orientación de los directivos en relación con el logro, el uso del tiempo, el cambio y la innovación, así como el riesgo (revelando su perspectiva ante el riesgo). (5) Liderazgo efectivo. Esto alude al estilo de gestión y capacidad de liderazgo para comunicar la visión estratégica (una visión clara del futuro de la organización) a los miembros de la organización y delegar poder a los empleados para llevarla a cabo, logrando su compromiso entusiasta.

En nuestro estudio, nos interesan los efectos directos de las competencias ejecutivas en sistemas de información (IS). Para ello, nos centramos en los aspectos específicos de las competencias ejecutivas en relación con la gestión de la información en las organizaciones, limitando nuestro estudio a las competencias ejecutivas en IS que se describen a continuación.

\section{CONCLUSIÓN}

Este estudio analiza las competencias que un gestor debe adquirir sobre los sistemas de información. Este documento muestra claramente la necesidad de que los gerentes medios o superiores logren estas competencias para lograr la ventaja competitiva en las organizaciones para las que trabajan. Una de las funciones más importantes de estas competencias es la posibilidad de crear una visión de TI y cómo estas tecnologías se pueden aplicar a las organizaciones. Siempre teniendo en cuenta esta visión estratégica, los cursos de sistemas de información para directivos deben desarrollar siete competencias esenciales. El primero es el conocimiento en tecnologías de la información. La segunda competencia está vinculada al conocimiento de las aplicaciones existentes en el mercado y qué programas existen para cada una de las funciones específicas de la organización y cómo pueden dar soporte a las actividades y a los procesos de toma de decisiones, así como a los sistemas de información como componente básico de coordinación entre actividades. En tercer lugar, es necesario crear competencias en el desarrollo y análisis de sistemas de información, vinculados a proyectos de creación o actualización de IS en las organizaciones. La cuarta competencia implica la adquisición de habilidades en la administración de IS y todos los aspectos relacionados con la seguridad y las auditorías. En quinto lugar, el comercio electrónico, la web 2.0 y las nuevas tendencias en tecnologías y aplicaciones móviles deben incluirse en los planes de estudio de las asignaturas de IS. En sexto lugar, los gerentes deben conocer el aspecto legal de la protección de datos, el comercio electrónico y el gobierno electrónico. Por último, aunque con un carácter menos académico, es necesario animar a los estudiantes sobre la importancia de conocer expertos y consultores de TI fiables que sean de vital importancia para las pymes. Este marco básico de competencias con el enfoque estratégico propuesto debe aparecer en las asignaturas de gestión de la información en titulaciones no técnicas con perfil directivo.

\section{TRANSLATED VERSION: FRENCH}

Below is a rough translation of the insights presented above. This was done to give a general understanding of the ideas presented in the paper. Please excuse any grammatical mistakes and do not hold the original authors responsible for these mistakes.

\section{VERSION TRADUITE: FRANÇAIS}

Voici une traduction approximative des idées présentées ci-dessus. Cela a été fait pour donner une compréhension générale des idées présentées dans le document. Veuillez excuser toutes les erreurs grammaticales et ne pas tenir les auteurs originaux responsables de ces erreurs.

\section{INTRODUCTION}

Le processus de Bologne exige la définition des objectifs à atteindre dans une matière en termes de connaissances, d'aptitudes et de compétences. Cela nécessite une réflexion approfondie sur les 
compétences, décrites en termes de connaissances, d'aptitudes, d'attitudes et d'aptitudes, que les diplômés doivent acquérir pour gérer avec succès leur travail, tant dans leurs tâches quotidiennes que dans leur développement professionnel tout au long de leur carrière. Développer ces compétences est l'objet final d'un programme éducatif (Commission européenne 2015).

Selon la Commission européenne (2015), la compétence peut être définie comme la capacité avérée d'utiliser les connaissances, les aptitudes et les capacités personnelles, sociales et/ou méthodologiques, dans des situations de travail ou d'études, et dans le développement professionnel et personnel.

Les compétences sont spécifiques à une matière ou génériques. Cet article vise à explorer les compétences spécifiques à la matière, en ce qui concerne les matières de système d'information qui sont enseignées dans les diplômes de gestion. Ces diplômes comprennent la gestion des affaires et l'administration publique. L'objectif de ces diplômes est de former des gestionnaires (cadres) capables d'assumer des responsabilités au sein des organisations pour lesquelles ils travaillent. Par conséquent, le présent document commence par définir le concept de compétence exécutive de manière générique, puis développe ces compétences exécutives en détail en ce qui concerne les systèmes d'information.

\section{Compétences Exécutives}

D’un point de vue commercial, la compétence est la capacité de soutenir le déploiement coordonné des actifs de manière à aider une entreprise à atteindre ses objectifs (Sanchez et al., 1996).

Les compétences exécutives sont essentielles, car elles permettent d'interpréter l'environnement et de créer une vision unique sur la base de laquelle cela est effectivement possible dans les affaires. Cette vision ou modèle mental (Senge 1990) de l'organisation et de l'environnement permet de détecter les opportunités et les menaces, établissant l'approche stratégique de l'organisation pour exploiter les asymétries sur les marchés avec des facteurs stratégiques (Lado et Wilson 1994; Hambrick et Mason, 1984). Les cadres sont les personnes chargées d'établir la mission et les objectifs qui guident la création et la mise en œuvre de la stratégie, ainsi que le développement des compétences et des capacités organisationnelles (Lado et al. 1992).

Les compétences exécutives proviennent à la fois de connaissances explicites et tacites, basées sur l'expérience des cadres. Les compétences exécutives comprennent généralement à la fois les capacités et le savoir-faire des cadres sur une base individuelle, ainsi que les compétences et les capacités découlant de l'interaction avec les autres membres du personnel.

Le leadership est également considéré comme un facteur de succès déterminant pour les gestionnaires. Le leadership permet de communiquer la mission et d'obtenir l'engagement de l'ensemble de l'organisation, permettant ainsi à ses membres d'agir de manière collective plutôt qu'isolée (Lado et Wilson 1994; Lado et coll., 1992; Westley et Mintzberg, 1989). Osbaldeston et Barham (1992) insistent sur ce point, indiquant le lien entre la compétitivité de l'entreprise et la compétence de gestion pour développer et utiliser complètement l'expérience et le talent de tous les membres de l'organisation, en intégrant et en encourageant les efforts d'une équipe complexe de personnes. Ainsi, les compétences en gestion couvrent des aspects aussi divers que les connaissances générales et spécialisées, les capacités individuelles ainsi que sociales, les compétences d'organisation et de planification, la capacité d'innover et de faire face à des activités non routinières.

Ces types de compétences déterminent de manière décisive l'acquisition, le développement et le déploiement des ressources et des capacités de l'entreprise, leur conversion en produits de valeur et la création de valeur. Ainsi, Penrose (1959), Hambrick et Brandon (1988) et Castanias et Helfat (1991) soulignent la valeur du talent des cadres et son hétérogénéité pour améliorer le rang concurrentiel de l'entreprise. Plus récemment, Bettiol et coll. (2012) confirment la valeur de la vision unique des gestionnaires d'interpréter l'environnement et l'importance de cette vision pour exploiter les ressources de l'entreprise (Yang et Li, 2011; Siegel et Renko, 2012).

Les chercheurs diffèrent par rapport aux dimensions qui composent les compétences exécutives. Par exemple, Lado et Wilson (1994: 703) distinguent uniquement deux dimensions: l'esquisse d'une vision stratégique et l'établissement d'un lien bénéfique avec l'environnement. Lado et al. (1992), cependant, identifient une troisième dimension, le leadership, indépendamment de la dimension de la vision 
stratégique. Camisón (2002) intègre ces modèles, en proposant cinq dimensions dans les compétences exécutives: (1) Approche stratégique. L'approche stratégique est la capacité de la direction à définir une vision stratégique qui appuie le développement des ressources organisationnelles et à établir un lien organisationnel et environnement bénéfique. (2) Qualification des cadres: Cela comprend leur talent, leurs connaissances techniques et de gestion, leur capacité à résoudre des problèmes et à prendre des décisions et leur expérience. (3) Personnalité des dirigeants. Cette dimension fait allusion à la connaissance des cadres sur eux-mêmes, à leur capacité à contrôler leur propre comportement et leurs émotions, ainsi qu'aux valeurs et aux normes qui les motivent, qui conditionnent leur modèle social et leur conduite. (4) Mentalité exécutive. Orientation des gestionnaires par rapport à la réussite, à l'emploi du temps, au changement et à l'innovation, ainsi qu'au risque (révélant leur point de vue face au risque). (5) Leadership efficace. Cela fait allusion au style de gestion et à la capacité de leadership de communiquer la vision stratégique (une vision claire de l'avenir de l'organisation) aux membres de l'organisation et de déléguer le pouvoir aux employés de la réaliser, en réalisant leur engagement enthousiaste.

Dans notre étude, nous nous intéressons aux effets directs des compétences exécutives dans les systèmes d'information (SI). À cette fin, nous nous sommes concentrés sur les aspects spécifiques des compétences exécutives en relation avec la gestion de l'information dans les organisations, en limitant notre étude aux compétences exécutives en SI qui sont décrites ci-dessous.

\section{CONCLUSION}

Cette étude analyse les compétences qu'un manager devrait acquérir sur les systèmes d'information. Ce document montre clairement la nécessité pour les cadres moyens ou supérieurs d'atteindre ces compétences afin d'obtenir l'avantage concurrentiel dans les organisations pour lesquels ils travaillent. L'une des fonctions les plus importantes de ces compétences est la possibilité de créer une vision de l'informatique et de la façon dont ces technologies peuvent être appliquées aux organisations. Toujours en gardant à l'esprit cette vision stratégique, les cours de système d'information pour les gestionnaires doivent développer sept compétences essentielles. Le premier est la connaissance des technologies de l'information. La deuxième compétence est liée à la connaissance des applications existantes sur le marché et aux programmes existants pour chacune des fonctions spécifiques de l'organisation et à la manière dont ils peuvent fournir un soutien aux activités et aux processus de prise de décision, ainsi que les systèmes d'information en tant que composante de coordination de base entre les activités. Troisièmement, il est nécessaire de créer des compétences dans le développement et l'analyse des systèmes d'information, liés à la création ou à la mise à niveau des SI dans les organisations. La quatrième compétence concerne l'acquisition de compétences dans l'administration des SI et tous les aspects liés à la sécurité et aux audits. Cinquièmement, le commerce électronique, le web 2.0 et les nouvelles tendances en matière de technologies et d'applications mobiles devraient être inclus dans les programmes des matières SI. Sixièmement, les gestionnaires doivent connaître l'aspect juridique de la protection des données, du commerce électronique et de l'administration en ligne. Enfin, bien qu'avec un caractère moins académique, il est nécessaire d'encourager les étudiants sur l'importance de connaître des experts et des consultants informatiques fiables qui sont d'une importance vitale pour les PME. Ce cadre de compétences de base avec l'approche stratégique proposée devrait apparaître dans les matières de gestion de l'information dans les diplômes non techniques avec un profil de gestion.

\section{TRANSLATED VERSION: GERMAN}

Below is a rough translation of the insights presented above. This was done to give a general understanding of the ideas presented in the paper. Please excuse any grammatical mistakes and do not hold the original authors responsible for these mistakes. 


\section{ÜBERSETZTE VERSION: DEUTSCH}

Hier ist eine ungefähre Übersetzung der oben vorgestellten Ideen. Dies wurde getan, um ein allgemeines Verständnis der in dem Dokument vorgestellten Ideen zu vermitteln. Bitte entschuldigen Sie alle grammatikalischen Fehler und machen Sie die ursprünglichen Autoren nicht für diese Fehler verantwortlich.

\section{EINLEITUNG}

Der Bologna-Prozess erfordert die Definition der Ziele, die in einem Fach in Bezug auf Kenntnisse, Fähigkeiten und Kompetenzen erreicht werden sollen. Dies erfordert ein gründliches Nachdenken über die Kompetenzen, die in Bezug auf Kenntnisse, Fähigkeiten, Einstellungen und Fähigkeiten beschrieben werden, die die Absolventen erwerben müssen, um ihre Arbeit sowohl in ihren täglichen Aufgaben als auch in ihrer beruflichen Entwicklung während ihrer gesamten Karriere erfolgreich zu bewältigen. Die Entwicklung dieser Kompetenzen ist das Endziel eines Bildungsprogramms (Europäische Kommission 2015).

Nach Angaben der Europäischen Kommission (2015) kann Kompetenz als die nachgewiesene Fähigkeit definiert werden, Kenntnisse, Fähigkeiten und persönliche, soziale und/oder methodische Fähigkeiten in Arbeits- oder Studiensituationen sowie in der beruflichen und persönlichen Entwicklung einzusetzen.

Kompetenzen sind fachspezifisch oder generisch. Dieses Papier zielt darauf ab, die fachspezifischen Kompetenzen in Bezug auf die Informationssystemfächer zu untersuchen, die in Management-Abschlüssen gelehrt werden. Diese Abschlüsse umfassen Betriebswirtschaft und öffentliche Verwaltung. Ziel dieser Abschlüsse ist es, Führungskräfte (Führungskräfte) auszubilden, die in der Lage sind, Verantwortung in den Organisationen zu übernehmen, für die sie arbeiten. Folglich beginnt dieses Papier mit der Definition des Konzepts der Exekutivkompetenz in einer generischen Weise und entwickelt anschließend diese Exekutivkompetenzen im Detail in Bezug auf Informationssysteme.

\section{Führungskompetenzen}

Aus geschäftlicher Sicht ist Kompetenz die Fähigkeit, den koordinierten Einsatz von Vermögenswerten auf eine Weise aufrechtzuerhalten, die einem Unternehmen hilft, seine Ziele zu erreichen (Sanchez et al. 1996).

Führungskompetenzen sind unerlässlich, da sie es ermöglichen, die Umwelt zu interpretieren und eine einzigartige Vision zu schaffen, auf deren Grundlage dies im Geschäft effektiv möglich ist. Diese Vision oder dieses mentale Modell (Senge 1990) der Organisation und der Umwelt ermöglicht es, Chancen und Bedrohungen zu erkennen und den strategischen Ansatz der Organisation zu etablieren, um Asymmetrien in Märkten mit strategischen Faktoren auszunutzen (Lado und Wilson 1994; Hambrick und Mason 1984). Führungskräfte sind die Personen, die für die Festlegung der Mission und der Ziele verantwortlich sind, die die Erstellung und Umsetzung der Strategie und die Entwicklung organisatorischer Fähigkeiten und Fähigkeiten leiten (Lado et al. 1992).

Die Führungskompetenzen entstehen sowohl aus explizitem als auch aus stillschweigendem Wissen, basierend auf den Erfahrungen der Führungskräfte. Die Führungskompetenzen umfassen in der Regel sowohl die Fähigkeiten und das Know-how der Führungskräfte auf individueller Basis als auch die Fähigkeiten und Fähigkeiten, die sich aus der Interaktion mit anderen Mitarbeitern ergeben.

Führung gilt auch als bestimmender Erfolgsfaktor für Führungskräfte. Führung ermöglicht es, die Mission zu kommunizieren und das Engagement der gesamten Organisation zu erhalten, so dass ihre Mitglieder kollektiv und nicht isoliert handeln können (Lado und Wilson 1994; Lado et al. 1992; Westley und Mintzberg 1989). Osbaldeston und Barham (1992) bestehen auf diesem Punkt und weisen auf die Verbindung der Wettbewerbsfähigkeit des Unternehmens mit der Managementkompetenz hin, um die Erfahrung und das Talent aller Mitglieder der Organisation zu entwickeln und vollständig zu nutzen, indem sie die Bemühungen eines komplexen Teams von Personen integrieren und fördern. So umfassen 
Managementkompetenzen so unterschiedliche Aspekte wie Allgemein- und Fachwissen, individuelle Fähigkeiten sowie soziale, Organisations- und Planungsfähigkeiten, Innovationsfähigkeit und die Bewältigung von nicht routinemäßigen Tätigkeiten.

Diese Art von Kompetenzen bestimmen entscheidend die Akquisition, Entwicklung und den Einsatz der Ressourcen und Kapazitäten des Unternehmens, deren Umwandlung in wertvolle Produkte und die Wertschöpfung. So betonen Penrose (1959), Hambrick und Brandon (1988) sowie Castanias und Helfat (1991) den Wert von Führungskräften und ihre Heterogenität, um den Wettbewerbsrang des Unternehmens zu verbessern. In jüngerer Zeit bestätigen Bettiol et al. (2012) den Wert der einzigartigen Vision der Manager, die Umwelt zu interpretieren, und die Bedeutung dieser Vision für die Nutzung der Ressourcen des Unternehmens (Yang und Li 2011; Siegel und Renko 2012).

Die Forscher unterscheiden sich in Bezug auf die Dimensionen, die die Exekutivkompetenzen umfassen. Zum Beispiel unterscheiden Lado und Wilson (1994: 703) nur zwei Dimensionen: die Umrisse einer strategischen Vision und die Herstellung einer vorteilhaften Verbindung mit der Umwelt. Lado et al. (1992) identifizieren jedoch eine dritte Dimension, die Führung, unabhängig von der Dimension der strategischen Vision. Camisón (2002) integriert diese Modelle und schlägt fünf Dimensionen in den Exekutivkompetenzen vor: (1) Strategischer Ansatz. Der strategische Ansatz ist die Fähigkeit der Führungskräfte, eine strategische Vision zu skizzieren, die die Entwicklung der organisatorischen Ressourcen unterstützt und eine vorteilhafte Verbindung zwischen Organisation und Umgebung herstellt. (2) Qualifizierung der Führungskräfte: Dazu gehören ihr Talent, ihr technisches und Managementwissen, ihre Fähigkeit, Probleme zu lösen und Entscheidungen zu treffen, sowie ihre Erfahrung. (3) Persönlichkeit der Führungskräfte. Diese Dimension spielt auf das Wissen der Führungskräfte über sich selbst, ihre Fähigkeit, ihr eigenes Verhalten und ihre Emotionen zu kontrollieren, und die Werte und Standards an, die sie motivieren, die ihr soziales Modell und Verhalten beeinflussen. (4) Exekutive Mentalität. Orientierung der Führungskräfte in Bezug auf Leistung, Zeitnutzung, Veränderung und Innovation sowie Risiko (Offenlegung ihrer Perspektive bei Risikobegesichtspunkten). (5) Effektive Führung. Dies spielt auf den Führungsstil und die Führungsfähigkeit an, die strategische Vision (eine klare Vision der Zukunft der Organisation) an die Mitglieder der Organisation zu kommunizieren und die Macht an die Mitarbeiter zu delegieren, um sie auszuführen und ihr begeistertes Engagement zu erreichen.

In unserer Studie interessieren wir uns für die direkten Auswirkungen der Führungskompetenzen in Wirtschaftsinformatik (IS). Zu diesem Zweck haben wir uns auf die spezifischen Aspekte der Führungskompetenzen in Bezug auf das Informationsmanagement in Organisationen konzentriert und unsere Studie auf die im Folgenden beschriebenen Führungskompetenzen in IS beschränkt.

\section{SCHLUSSFOLGERUNG}

Diese Studie analysiert die Kompetenzen, die ein Manager über Informationssysteme erwerben sollte. Dieses Papier zeigt deutlich, dass mittlere oder obere Manager diese Kompetenzen erreichen müssen, um den Wettbewerbsvorteil in den Organisationen zu erreichen, für die sie arbeiten. Eine der wichtigsten Funktionen dieser Kompetenzen ist die Möglichkeit, eine Vision der IT zu erstellen und wie diese Technologien auf Organisationen angewendet werden können. Immer unter Berücksichtigung dieser strategischen Vision müssen die Informationssystemkurse für Führungskräfte sieben wesentliche Kompetenzen entwickeln. Das erste ist das Wissen über Informationstechnologien. Die zweite Kompetenz ist mit der Kenntnis der bestehenden Anwendungen auf dem Markt verbunden und welche Programme für jede der spezifischen Funktionen der Organisation existieren und wie sie die Aktivitäten und die Entscheidungsprozesse sowie die Informationssysteme als grundlegende Koordinationskomponente zwischen den Aktivitäten unterstützen können. Drittens ist es notwendig, Kompetenzen in der Entwicklung und Analyse von Informationssystemen zu schaffen, die mit IS-Erstellungs- oder Upgrade-Projekten in den Organisationen verbunden sind. Die vierte Kompetenz umfasst den Erwerb von Fähigkeiten in der ISVerwaltung und alle Aspekte, die mit Sicherheit und Audits verbunden sind. Fünftens sollten E-Commerce, Web 2.0 und die neuen Tendenzen bei mobilen Technologien und Anwendungen in die Lehrpläne der ISFächer aufgenommen werden. Sechstens müssen Manager über den rechtlichen Aspekt von Datenschutz, 
E-Commerce und E-Government Bescheid wissen. Schließlich, obwohl mit einem weniger akademischen Charakter, ist es notwendig, studenten zu ermutigen, wie wichtig es ist, zuverlässige IT-Experten und Berater zu kennen, die für KMU von entscheidender Bedeutung sind. Dieser grundlegende Kompetenzrahmen mit dem vorgeschlagenen strategischen Ansatz sollte in den Fächern Informationsmanagement in nicht-technischen Abschlüssen mit einem Managementprofil erscheinen.

\section{TRANSLATED VERSION: PORTUGUESE}

Below is a rough translation of the insights presented above. This was done to give a general understanding of the ideas presented in the paper. Please excuse any grammatical mistakes and do not hold the original authors responsible for these mistakes.

\section{VERSÃO TRADUZIDA: PORTUGUÊS}

Aqui está uma tradução aproximada das ideias acima apresentadas. Isto foi feito para dar uma compreensão geral das ideias apresentadas no documento. Por favor, desculpe todos os erros gramaticais e não responsacule os autores originais responsáveis por estes erros.

\section{INTRODUÇÃO}

O processo de Bolonha exige a definição dos objetivos a serem alcançados em um assunto em termos de conhecimento, habilidades e competências. Isso requer um pensamento aprofundado sobre as competências, descritas em termos de conhecimento, habilidades, atitudes e habilidades, nas quais os graduados devem adquirir para lidar com sucesso seu trabalho, tanto em suas tarefas diárias quanto em seu desenvolvimento profissional ao longo de sua carreira. Desenvolver essas competências é o objetivo final de um programa educacional (Comissão Europeia 2015).

Segundo a Comissão Europeia (2015), a competência pode ser definida como a capacidade comprovada de utilizar conhecimentos, habilidades e habilidades pessoais, sociais e/ou metodológicas, em situações de trabalho ou estudo, e no desenvolvimento profissional e pessoal.

As competências são específicas ou genéricas. Este artigo tem como objetivo explorar as competências específicas do assunto, em relação aos sujeitos do sistema de informação que são ensinados em cursos de gestão. Esses cursos incluem Gestão empresarial e Administração Pública. O objetivo desses diplomas é formar gestores (executivos) capazes de assumir a responsabilidade dentro das organizações para as quais atuam. Consequentemente, este artigo começa definindo o conceito de competência executiva de forma genérica, posteriormente, desenvolvendo essas competências executivas em detalhes em relação aos sistemas de informação.

\section{Competências Executivas}

Do ponto de vista empresarial, a competência é a capacidade de sustentar a implantação coordenada de ativos de forma que ajude uma empresa a alcançar seus objetivos (Sanchez et al. 1996).

As competências executivas são essenciais, pois permitem interpretar o meio ambiente e criar uma visão única baseada na qual é efetivamente possível nos negócios. Essa visão ou modelo mental (Senge 1990) da organização e do meio ambiente possibilita detectar oportunidades e ameaças, estabelecendo a abordagem estratégica da organização para explorar assimmetrias em mercados com fatores estratégicos (Lado e Wilson 1994; Hambrick e Mason 1984). Os executivos são os responsáveis pela definição da missão e objetivos que norteiam a criação e implementação da estratégia, e o desenvolvimento de habilidades e capacidades organizacionais (Lado et al. 1992).

As competências executivas são originárias do conhecimento explícito e tácito, a partir da experiência dos executivos. As competências executivas geralmente incluem tanto as habilidades quanto o know-how dos executivos de forma individual, bem como as habilidades e capacidades derivadas da interação com outros membros da equipe. 
A liderança também é considerada como um fator determinante de sucesso para os gestores. A liderança permite comunicar a missão e obter o compromisso de toda a organização, permitindo assim que seus membros atuem de forma coletiva e não isolada (Lado e Wilson 1994; Lado et al. 1992; Westley e Mintzberg 1989). Osbaldeston e Barham (1992) insistem neste ponto, indicando o vínculo da competitividade da empresa com a competência de gestão para desenvolver e utilizar completamente a experiência e o talento de todos os membros da organização, integrando e incentivando os esforços de uma equipe complexa de pessoas. Assim, as competências de gestão abrangem aspectos tão diversos quanto conhecimentos gerais e especializados, habilidades individuais, bem como habilidades sociais, organização e planejamento, capacidade de inovar e lidar com atividades não rotineiras.

Esses tipos de competências determinam decisivamente a aquisição, desenvolvimento e implantação dos recursos e capacidades da empresa, sua conversão em produtos valiosos e a criação de valor. Assim, Penrose (1959), Hambrick e Brandon (1988), e Castanias e Helfat (1991) enfatizam o valor do talento executivo e sua heterogeneidade para melhorar o ranking competitivo da empresa. Mais recentemente, Bettiol et al. (2012) confirmam o valor da visão única dos gestores para interpretar o meio ambiente, e a importância dessa visão para explorar os recursos da empresa (Yang e Li 2011; Siegel e Renko 2012).

Os pesquisadores diferem em relação às dimensões que compõem as competências executivas. Por exemplo, Lado e Wilson (1994: 703) distinguem apenas duas dimensões: o esboço de uma visão estratégica e o estabelecimento de um elo benéfico com o meio ambiente. Lado et al. (1992), no entanto, identificam uma terceira dimensão, liderança, independentemente da dimensão da visão estratégica. Camisón (2002) integra esses modelos, propondo cinco dimensões nas competências executivas: (1) Abordagem estratégica. A abordagem estratégica é a capacidade executiva de traçar uma visão estratégica que apoie o desenvolvimento dos recursos organizacionais e estabeleça um elo de organização e meio ambiente benéfico. (2) Qualificação dos executivos: Isso inclui seu talento, conhecimento técnico e gerencial, sua capacidade de resolver problemas e tomar decisões e sua experiência. (3) Personalidade dos executivos. Essa dimensão alude ao conhecimento dos executivos sobre si mesmos, à sua capacidade de controlar seu próprio comportamento e emoções, e aos valores e padrões que os motivam, que condicionam seu modelo social e conduta. (4) Mentalidade executiva. Orientação dos gestores em relação à realização, uso do tempo, mudança e inovação, bem como risco (revelando sua perspectiva ao enfrentar riscos). (5) Liderança eficaz. Isso alude ao estilo de gestão e à capacidade de liderança de comunicar a visão estratégica (uma visão clara do futuro da organização) aos membros da organização e delegar poder aos colaboradores para realizá-la, alcançando seu compromisso entusiasmado.

Em nosso estudo, estamos interessados nos efeitos diretos das competências executivas nos sistemas de informação (IS). Para isso, focamos nos aspectos específicos das competências executivas em relação à gestão da informação nas organizações, limitando nosso estudo às competências executivas do EI que estão descritas abaixo.

\section{CONCLUSÃO}

Este estudo analisa as competências que um gestor deve adquirir sobre sistemas de informação. Este artigo mostra claramente a necessidade de gestores médios ou superiores alcançarem essas competências para alcançar a vantagem competitiva nas organizações para as quais trabalham. Uma das funções mais importantes dessas competências é a possibilidade de criar uma visão de TI e como essas tecnologias podem ser aplicadas às organizações. Sempre tendo essa visão estratégica em mente, os cursos do sistema de informação para gestores devem desenvolver sete competências essenciais. O primeiro é o conhecimento em tecnologias da informação. A segunda competência está vinculada ao conhecimento das aplicações existentes no mercado e quais programas existem para cada uma das funções específicas da organização e como podem dar suporte às atividades e aos processos decisórios, bem como aos sistemas de informação como componente básico de coordenação entre as atividades. Em terceiro lugar, é necessário criar competências no desenvolvimento e análise de sistemas de informação, vinculados à criação ou atualização de projetos do EI nas organizações. A quarta competência envolve a aquisição de habilidades na administração do EI e todos os aspectos ligados à segurança e auditorias. Em quinto lugar, o e-commerce, 
o web 2.0 e as novas tendências em tecnologias e aplicações móveis devem ser incluídos nos currículos dos sujeitos do EI. Em sexto lugar, os gestores devem conhecer o aspecto legal da proteção de dados, ecommerce e e-governo. Finalmente, embora com uma natureza menos acadêmica, é necessário incentivar os alunos sobre a importância de conhecer especialistas e consultores confiáveis de TI que são de vital importância para as PME. Esse quadro básico de competências com a abordagem estratégica proposta deve aparecer nos temas da gestão da informação em graus não técnicos com perfil de gestão. 